\title{
Brain Cancer Investigation with Interfacing of 3-Dimensional Image Processing
}

\author{
Soobia Saeed ${ }^{1 *}$, Afnizanfaizal Abdullah' and NZ. Jhanjhi ${ }^{2}$ \\ 'Department of Software Engineering, Universiti Teknologi Malaysia-UTM, Johor Bharu, Malaysia; Saeed.soobia@ \\ graduate.utm.my, afnizanfaizal@utm.my \\ ${ }^{2}$ School of Computing and IT (SoCIT), Taylor's University, Subang Jaya, Selangor, Malaysia; noorzaman.jhanjhi@ \\ taylors.edu.my
}

\begin{abstract}
Background/Objectives: 3D (3-dimensional) image analysis (3-D) provides an effective way to quickly and accurately evaluate complex interactions and functions between neurons. Method of identification based on the neuronal program. Other computerized image processing frameworks, for example, the corresponding schemes of data generate similar rays. For the most portions, on the same side of the heated head (heat shock proteins) the problem of brain tissue due to glucose is more than the tissue on the other side of the brain. Causes of brain tumors (cancer) are still unknown in this research owing to the rise in glucose metabolism. Methods/Findings: Our study region focuses on slowly developing paper tumors and oral tumors on the side of the brain. This research is the extended version of the research presented in (ICISCT), 2019. The aim of the research is to tackle the above-mentioned brain tissue issues. Application: Our study focuses on how to reduce the effect of cancer and boost human life through chemotherapy and how to define the process of 3-dimensional segmentation. The author operates on brain cancer in this study job and applies a statistical model to the trials and discusses pictures of brain tumors produced using MATLAB software. It then describes the solution from the medical point of view and application and predicts the future of the modified technology.
\end{abstract}

Keywords: Brain tumor, Cancer, Dimensional, Heat shock proteins, Process, Segmentation, Tissues

\section{Introduction}

Helpful images give critical information on human wellbeing. In the system of image investigation, image fracture is one of the fundamental subjects, maybe the most talked about in the writing. The techniques for image discontinuity were the subject of a few research works. Reviews on existing methodologies can be found, for instance, in $\frac{1-5}{}$. Image fracture is typically the initial step before applying any an algorithm to envision the PC, for example, protest acknowledgement. The chipping procedure comprises of amassing image components, known as pixels or voxels (for two-and three-dimensional images, separately), into homogeneous districts called locales. Every district is bound together in connection to particular properties dependent on thickness (grey levels), texture or colours. The area group is a portion of the image, so any pixel or voxel in the image is completely confined to an area. There are many fields of science and engineering where three-dimensional data (3-D) are collected and analyzed, such as medical imagery and geoscience. To design and test the validity of computational procedures to process and analyze these data, the need for computational theory and algorithms for image processing is essential ${ }^{6-9}$. Three-dimensional images (3-D) and visualization have been the subject of many types of research because of their various advantages and applications $\frac{10,11}{}$. However,

*Author for correspondence 
because of the need to capture, record, process and display a large amount of visual data to produce high-quality $3 \mathrm{D}$ images, the 3D imaging techniques developed have had to affect their actions or the use of special devices and techniques. As coherent incisions, special glasses, which is the nuisance of the most practical operation ${ }^{12-14}$ ?

This technique is a kind of three-dimensional imaging system in multiple views that uses a series of diffraction or refractive elements to capture optical data in 3D. It has recently attracted considerable attention since it produces automatic images of self-regulation without special lighting requirements. However, a traditional system cannot produce high-resolution 3D images, large field depth, and a large viewing angle. This document provides an overview of approaches and techniques developed over the last decade to overcome these constraints. By integrating these technologies with the looming technology, 3D image systems are expected to achieve practical application in various fields $s^{15-17}$.

A tumor is described as the unpredictable improvement of the tissues. The brain tumor is an anomalous mass of tissue in which cells build up, what's more, copy uncontrollably, clearly unchecked by the parts that control ordinary cells. The cell can be evaluated as the key fundamental unit of each and every living thing. The human body contains around 100 trillion cells and every one of them has its individual capacities with regards to the most ideal working of the body, these cells need to crevice to shape new cells controllably ${ }^{18-22}$. In any case, once in a while, they segment and wind up being fiercely to diagram new cells. This outcome in a square of undesirable tissue which is depicted as a tumor. Tumors can occur in many parts of the body. A brain tumor can be assumed as one of the honest to goodness and lifecrippling tumors. Tumors are portrayed considering the territory of their beginning and its dangers $23-32$.

\section{Mathematical Model of Tumor}

The tumor has some steps and each phase acknowledges the index of the tumor from the primary to the tumor's bottom level. We also acknowledge that the researched meningioma and glioma called glioblastomamultiforme are the most prevalent species in adolescents. Some species are unusually uncommon. Brain tumors may happen at any age. For instance, in adolescents, a few types of medulloblastoma are more common, and in adults some are more fundamental. Overall, with growing age, tumors that appear in adults become more regular. Brain tumors are distributed more uniformly than tumors of the brain that are damaging and malignant. The first indicators may include an acronym for migraine and a sensation. These are due to the extra weight inside the skull and the weight inside the skull. These indicators can come and go from the beginning and tend to be more unfortunate in the morning. Penetration, breathing and tendency can exacerbate brain pain. Epileptic seizures, for example, sometimes heal. Expand the language, for example. As the tumor increases, there may be a tendency to laziness and laziness. As the tumor develops, it can damage the nearby brain tissue. Different body parts are governed by separate brain components. Subsequently, the indices differ from case to case depending on the portion of the brain and the magnitude of the region impacted. For instance, one or more friends in an arm, leg or piece of face or eyes can generate muscle weakness, displacement issues, joint appointment, vision, hearing, voice, correspondence or swallowing, Loss of smell, dizziness that creates happiness, confusion or weakness, numbness or lack of one part of the body, confusion, personality changes and symptoms that have been identified by hormonal changes in case you have a pituitary tumor. If there is a suspected brain tumor due to side effects, a specialist will inspect them. This will involve looking out for the development of the brain and nerves, reflexes, vision, etc. capabilities. The result of MRI or CT scans of the head is regular tests performed to confirm or exclude near brain tumor. (See separate manuals called MRI output and computed tomography for more precise elements.) If there is a distinct tumor, more tests and tests can be performed from point to point. In this case, a PET scan or angiography is performed to obtain more information about the tumor. It is anticipated that the biopsy will determine the tumor type. A biopsy is a point where a tiny tissue sample is expelled from a body portion. The sample is then examined to look for abnormal cells under a magnifying glass. A tiny surgery 
generally needs to be done using a sedative for a brain tumor biopsy. A small area of the skull was mined to allow for the accurate needle to pass through a small example of tissue. To control the cells acquired by biopsy, the type of tumor can be distinguished and, if threatened, can assess the tumor.

\section{Methodology}

The main objective of our work is to develop a system that can detect the CSF leakage and tumors region or can separate between tumors and non-tumors patient. Initially, the input MRI image is $4 \mathrm{D}$-Light Field Tool (LFT) segmentation in order to fix the image for rest of th. The proposed system is based on the tumor and its computation, calculated using MATLAB (a threedimensional segmentation process). In this exploration, the researcher works away at the tumor and its developing size and ascertains the area of the local part by choosing an alternate tumor measure that develops well-ordered around then (around 5 to 10 years). These means demonstrate the size and meaning of the age of the tumor, and the quantity of years/months/weeks in which people live with the tumor.

\section{Algorithm}

Step 1: Input: Load MRI Image (MRI)

Step 2. Get Image (MRI $)$

Step 3: Input: Load MRI Image $\left(\mathrm{MR}_{2}\right)$

Step 4. Get Image $\left(\mathrm{MRI}_{2}\right)$

Step 5: images $\left(M^{*} \mathrm{~N}\right)$

Step 6. Select ROI Of $\mathrm{I}_{1} \& \mathrm{I}_{2}$.

Step 7: Step.10: Applying Gaussian Filter to unsharp Filters

STEP 8: Save images MRI and $\mathrm{H}_{1}$

STEP 9: Compare Filtered Images with Original

Images

Img1 = image subtract $\left(\mathrm{MRI}_{\mathrm{F} 1}, \mathrm{MR}_{1}\right)$

$\mathrm{K} 1$ = Inverse $(\operatorname{Img} 1)$;

SHOW Image (K1)

SHOW Image (MRI)

Img2 = image subtract $\left(\mathrm{MRI}_{\mathrm{F} 2}, \mathrm{MRI}_{2}\right)$
K2 = Inverse (Img2);

SHOW Image (K2)

SHOW Image $\left(\mathrm{MRI}_{2}\right)$

Step 10: Isize $=$ Size of the Image

$\mathrm{B}=$ Block Size i.e. $2,4,8$ etc

$\mathrm{P}[255]=$ COMPUTE the Maximum Occurrence Pixel Value

Iseg $[B][1024]=$ Segment of Image Read Each Time

Begin

Step 11: Open MRI file.

Step 12: Open $\mathrm{MRI}_{2}$ file.

Step 13: Open HI file.

Step 14: Loop J = 0, Isize

SAVE MRI $_{\mathrm{F} 1}$ and MRI $\mathrm{F}_{\mathrm{F}}$

COMPUTE

Result $=$ Number of Non Zero (ExtDiff)

COMPUTE PERCENTAGE

$[\mathrm{M}, \mathrm{N}]=$ Size of $\operatorname{Img}($ Result $)$

Percent $=\left(\text { Result } /\left(M^{*} \mathrm{~N}\right)\right)^{*} 100$

ELSE

SAVE $\mathrm{MR}_{1}$ and $\mathrm{MRI}_{2}$ COMPUTE

Result $=$ Number of Non Zero (ExtDiff)

COMPUTE PERCENTAGE

$[\mathrm{M}, \mathrm{N}]=$ Size of $\operatorname{Img}($ Result $)$

Percent $=($ Result $/(M * N)) * 100$

Step 15: SHOW IMAGES

COMPUTE the difference

\section{Result and Discussion}

In this research article, the researcher analyzes the detecting of a brain cancer due to interfacing of MRI-3D Images. It is a qualitative research study. Our aim of this research is to construct a proposed framework that can identify cancer damage area or be isolated from tumors and non-tumors quiet. Initially, the MRI processed the pre-processed image method with the final target selected to adjust the image for the rest of the procedures. On the basis of this study is the detection of brain cancer due to the process of interfacing the $4 \mathrm{D}$ image segmentation process are Figure 1. 


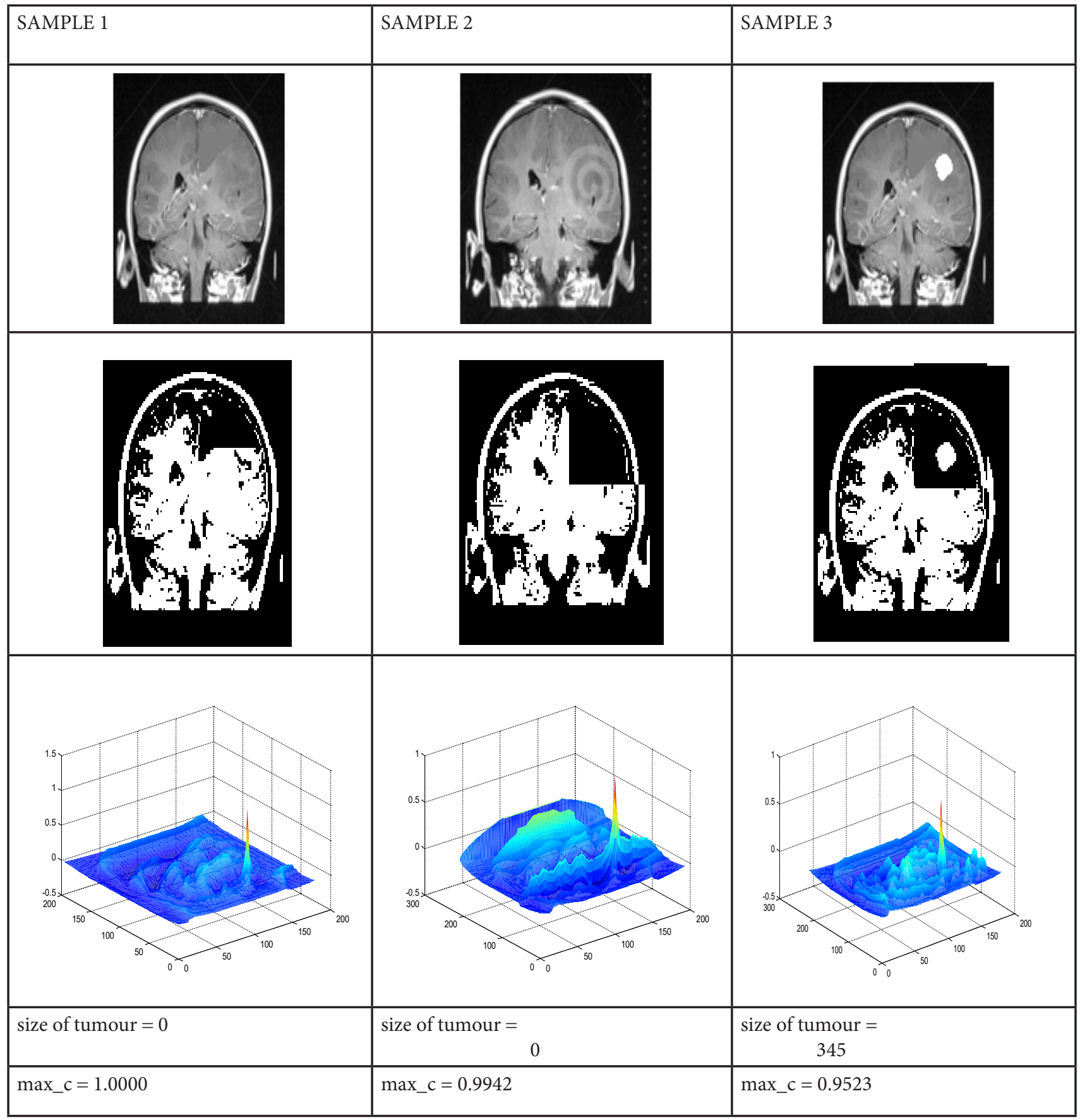

Figure 1. Images of detection of brain tumor.

Therefore, it consists of primary and secondary sources after a research study, followed by MATLAB modeling techniques with the use of original medical sample images to measure the range of brain damage cells deep inside of brain. To image segmentation process by using MATLAB Algorithm. In this study, the researcher proposes a 3D modulation method that supervises the machine learning that can be used by $2 \mathrm{D}$ to $3 \mathrm{D}$ segmentation method. The researcher uses the technique for editing the brain skull damaged brain samples. These findings show the effectiveness of our approach to light editing applications. These light field methods can be useful for improving the quality of the segmentation of application editing, as they reduce boundary artefacts. 
The researcher evaluated the methods for overcoming the computational experiments of the proposed new method. We use the algorithm with correlation and multiple data types in time series data. We made it clear that the improvement of the calculated data to determine the validation method variables is associated with the time delay of the test and the training vector resulting from the time delay.

\section{Conclusion}

This research demonstrates brain tumor using brain tumor segmentation (3-dimensional segmentation) between pictures of MRI and displaying the outcomes of a newly suggested algorithm. For instance, magnetic resonance imaging, PET or figured tomography, the real medicinal pictures direct an extremely boggling BRAIN examination due to privacy problems and actual specific snags. The motive behind this experiment is to dissect methods to identify brain tumors through MRI, obtain MRI data from the internet database of the brain, and show a image of the brain's MRI test. In this study job, the investigator operates on these tumor-dependent pictures and is using three-dimensional image sections to apply the segment region to the tumor.

These images mainly represent tumor size and need to figure tumor measure with the assistance of MATLAB. In this paper, we center on tumor measure and compute zone from venture to venture of last tumor cell development. Utilizing an alternate MATLAB device, we have tried in excess of multiple skull tests that uncover the area of the cerebrum tumor and furthermore apply its very own soundness. Cerebrum tumors are the fundamental territory of our exploration. Accuracy is the key device for progress, so this investigation recommends MRI to get the best images and best outcomes.

\section{Acknowledgements}

Authors are grateful to the Department of Software Engineering, faculty of computing, Universiti Teknologi Malaysia-UTM, Malaysia for financial support to carry out this work.

\section{References}

1. Levman J, Takahashi E. Pre-adult MRI of brain cancer and neurological injury: Multivariate analyses. Front Pediatr. 2016; 4:65. https://doi.org/10.3389/fped.2016.00065 PMid:27446888 PMCid:PMC4917540

2. Koob M, Girard N, Ghattas B. The diagnostic accuracy of multiparametric MRI to determine pediatric brain tumor grades and types. Journal of Neuro-Oncology. 2016; 127(2):345-53. https://doi.org/10.1007/s11060-015-2042-4 PMid:26732081

3. SchneiderJF,Confort-GounyS, ViolaA, etal.Multiparametric differentiation of posterior fossa tumors in children using diffusion-weighted imaging and short echo-time $1 \mathrm{H}-\mathrm{MR}$ spectroscopy. Journal of Magnetic Resonance Imaging. 2007; 26(6):1390-8. https://doi.org/10.1002/jmri.21185 PMid:17968955

4. Reynolds GM, Peet AC, Arvanitis TN. Generating prior probabilities for classifiers of brain tumours using belief networks. BMC Medical Informatics and Decision Making. 2007; 7:27. https://doi.org/10.1186/1472-6947-7-27 PMid:17877822 PMCid:PMC2040142

5. Haney S, Thompson P, Cloughesy T, Alger J, Toga A. Tracking tumor growth rates in patients with malignant gliomas: A test of two algorithms. American Journal of Neuroradiology. 2001; 22(1):73-82.

6. Swanson K, Alvord E, Murray J. Virtual brain tumours (gliomas) enhance the reality of medical imaging and highlight inadequacies of current therapy. British Journal of Cancer. 2002; 86(1):14-8. https://doi.org/10.1038/ sj.bjc.6600021 PMid:11857005 PMCid:PMC2746525

7. Xia Y, Bettinger K, Shen L, Reiss A. Automatic segmentation of the caudate nucleus from human brain MR images. IEEE Transactions on Medical Imaging. 2007; 26(4):509-17. https://doi.org/10.1109/TMI.2006.891481 PMid:17427738

8. Chu C, Takaya K. 3-Dimensional rendering of MR images. IEEE WESCANEX 93 Communications, Computers and Power in the Modern Environment- Conference Proceedings; 2003. p. 165-70.

9. Clarke L, Velthuizen R, Camacho M, Heine J, Vaydianathan M, Hall L, Thatcher R, Silbiger M. MRI segmentation: Methods and applications. Magnetic Resonance Imaging. 2013; 1-12.

10. Cline H, Lorensen W, Kikinis R, Jolesz F. Three-dimensional segmentation of MR images of the head using probability and connectivity. International Journal of Computer Science and Engineering Survey (IJCSES). 200; 6(6):1-16. 
11. Eisenhauer E. New response evaluation criteria in solid tumours: Revised RECIST guideline (version 1.1). European Journal of Cancer. 2009; 45(2):228-47. https://doi.org/10.1016/j.ejca.2008.10.026 PMid:19097774

12. Wen PY. Updated response assessment criteria for highgrade gliomas: Response assessment in a neuro-oncology working group. Journal of Clinical Oncology. 2010; 28:1963-72. https://doi.org/10.1200/JCO.2009.26.3541 PMid:20231676

13. Angelini ED, Clatz O, Mandonnet E, Konukoglu E, Capelle L, Duffau H. Glioma dynamics and computational models: A review of segmentation, registration, and in silico growth algorithms and their clinical applications. Current Medical Imaging Reviews. 2017; 3:262-76. https://doi. org/10.2174/157340507782446241

14. Saeed S, Raza Naqvi SM. Detection of brain cancer using MATLAB techniques. Journal of Medical imaging and Health Informatics (JMIHI). 2016; 7(6):1454-60. https://doi.org/10.1166/jmihi.2017.2187

15. Saeed S. Technique for tumour detection upon brain MRI Image by utilizing Support Vector Machine. Quaid-EAwam University of Engineering, Science and Technology. 2018; 16(1):37-42.

16. Saeed S, Ahmed Noor S. Analysis of brain cancer due to the usage of mobile phone. Mehran University of Engineering Journal (MUET). 2017; 36(3):609-20. https://doi.org/10.22581/muet1982.1703.17

17. Saeed S. Estimation of cerebrum cancer using latest technology of mobile phone. Journal of Information and Communication Technology. 2015; 9(1):23-32.

18. Weizman L. Automatic segmentation, internal classification, and follow-up of optic pathway gliomas in MRI. Med Image Anal. 2012; 16(1):177-88. https://doi.org/10.1016/ j.media.2011.07.001 PMid:21852179

19. Styner M. 3D segmentation in the clinic: A grand challenge II: MS lesion segmentation. Migraine Disability Assessment. 2008; 1-5.

20. Geremia E, Clatz O, Menze BH, Konukoglu E, Criminisi A, Ayache N. Spatial decision forests for MS lesion segmentation in multi-channel magnetic resonance images. Neuroimage. 2011; 57:378-90. https://doi.org/10.1016/j. neuroimage.2011.03.080 PMid:21497655

21. Geremia E, Menze BH, Ayache N. Spatially adaptive random forests. IEEE International Symposium on Biomedical Imaging. 2013; 1344-7. https://doi.org/10.1109/ ISBI.2013.6556781

22. Bauer S, Nolte LP, Reyes M. Fully automatic segmentation of brain tumor images using support vector machine classification in combination with hierarchical conditional random field regularization. International Conference on Medical Image Computing and Computer-Assisted
Intervention; 2011. p. 354-61. https://doi.org/10.1007/9783-642-23626-6_44 PMid:22003719

23. Riklin-Raviv T, Van Leemput K, Menze BH, Wells WM, Golland P. Segmentation of image ensembles via latent atlases. Medical Image Analysis. 2010; 14:654-65. https://doi.org/10.1016/j.media.2010.05.004 PMid:20580305 PMCid:PMC2932709

24. Cobzas D, Schmidt M. Increased discrimination in level set methods with embedded conditional random fields. Proceedings of the IEEE conference on computer vision and pattern Recognition (CVPR); 2009. p. 328-35. https://doi.org/10.1109/CVPRW.2009.5206812

25. Corso J. Efficient multilevel brain tumor segmentation with integrated Bayesian model classification. IEEE Transactions on Medical Imaging. 2008; 27(5):629-40. https://doi. org/10.1109/TMI.2007.912817 PMid:18450536

26. Gooya A. GLISTR: Glioma image segmentation and registration. IEEE Transactions on Medical Imaging. 2012; 31(10):1941-54.https://doi.org/10.1109/TMI.2012.2210558 PMid:22907965 PMCid:PMC4371551

27. Parisot S, Duffau H, Chemouny S, Paragios N. Joint tumor segmentation and dense deformable registration of brain MR images. International Conference on Medical Image Computing and Computer-Assisted Intervention; 2012. p. 651-8. https://doi.org/10.1007/978-3-642-33418-4_80 PMid:23286104

28. Clatz O, Delingette H, Bardinet E, Dormont D, Ayache N. Patient-specific biomechanical model of the brain: Application to Parkinson's disease procedure. International Symposium on Surgery Simulation and Soft Tissue Modeling; 2003. p. 321-31. https://doi.org/10.1007/3-54045015-7_31

29. Pohl KM, Fisher J, Levitt JJ, Shenton ME, Kikinis R, Grimson W, Wells WM. A unifying approach to registration, segmentation, and intensity correction. International Conference on Medical Image Computing and Computer-Assisted Intervention; 2005. p. 310-8. https://doi.org/10.1007/11566465_39 PMid:16685860

30. Geremia E, Menze BH, Ayache N. Spatially adaptive random forests. Proceedings IEEE International Symposium on Biomedical Imaging; 2013. p. 1344-7. https://doi. org/10.1109/ISBI.2013.6556781

31. Mihara H, Funatomi T, Tanaka K, Kubo H. 4D light field segmentation with spatial and angular consistencies. Japan: MEXT; 2018. p. 1-8.

32. Saeed $S$, Abdullah AB. Investigation of a Brain Cancer with Interfacing of 3-Dimensional Image Processing. International Conference on Information Science and Communication Technology (ICISCT); Karachi, Pakistan. 2019. p. 1-6. 